\title{
An investigation of the nature of abstract concepts
}

\author{
JAMES A. HAMPTON \\ City University, London ECIV OHB, England
}

\begin{abstract}
Eight abstract concepts were submitted to a procedure designed to test the logical nature of their definition. The hypothesis that they would show a polymorphous prototype structure similar to that found for concrete categories (Hampton, 1979; Rosch \& Mervis, 1975) was confirmed for five of the concepts. Reasons for the lack of fit of the prototype model to the remaining concepts and implications for the generality of existing theories of semantic memory are discussed.
\end{abstract}

Recent work in semantic memory and related fields has given considerable insight into the structure of natural concepts. In particular, the work of Rosch (e.g., Rosch \& Mervis, 1975) has shown how natural categories such as "fruit" or "furniture" have an internal structure centered around a prototype. Thus some members of such a category are close to the prototypical form, or center of the category. Compared with more peripheral items, these category members are consistently rated as more typical (Rosch, 1975), are categorized faster (Hampton, 1979; Smith, Shoben, \& Rips, 1974), and share more properties in common with other category members (Rosch \& Mervis, 1975).

Hampton (1979) described the structure of these categories of concrete nouns as "polymorphous concepts." A polymorphous concept is one in which an instance belongs to a certain category if and only if it possesses a sufficient number of a set of features, none of which need be common to all category members. For example, "sweetness" is a feature of fruit, but several important members of the category (such as lemons) do not possess it. Thus there may be many features that are neither necessary (required for membership) nor sufficient (guaranteeing membership) but yet are important to the category definition (other examples are "growing on trees," "roundness," and "having peel"). The procedure adopted by Hampton (1979) for testing the polymorphous model involved examining the relationship between the featural definition of a concept and the rated typicality of its exemplars. Subjects were asked to provide featural definitions for eight categories. Thus, for example, they were asked to name those charac-

This research was supported by a postgraduate studentship awarded to the author at University College London by the Medical Research Council of Great Britain. The author wishes to thank David Green for his help and guidance in the research and Peter Fonagy and John Gardiner for their comments on earlier drafts of this paper. Requests for reprints should be sent to James A. Hampton, Department of Social Science and Humanities, City University, Northampton Square, London EC1V OHB, England. teristics that are generally important for deciding if an object is a kind of fruit, and the most commonly given properties were then treated as featural definitions for that category. A list of words, including category members, were then rated in two ways: first, for the degree to which they belonged to the category, and second (by an independent group of judges), for the extent to which they possessed each of the category features. It was found, as predicted by the polymorphous concept model, that the number of category features that a word possessed predicted the rated degree of membership of the word in a category, the number of people making a "yes" or "no" category decision about the word, and the speed with which these decisions were made. Perhaps most important, for the majority of the categories, no common-feature type of definition was possible. The categories could therefore not be given a conjunctive definition, as required, for example, by the model of Smith et al. (1974).

Further work by McCloskey and Glucksberg (1979) supports the notion that natural categories are structured as polymorphous concepts. They provide evidence for a category decision process based on a Bayesian decision model, in which sequential feature comparisons between an item and a category are undertaken to provide evidence for and against a positive reponse. This model, named the property comparison model, is designed to operate on concepts which are polymorphous collections of features of different importance or salience.

The question to which the present paper is addressed concerns the generality of this set of models to other kinds of concepts. In particular, can it be shown that more abstract and culturally relevant concepts such as "art" or "work" can be defined as polymorphous concepts?

No attempts have been made previously to generalize semantic memory models to apply to abstract concepts, and as a result very little is known about their structure. One reason for this state of ignorance may be that certain models, such as network or spreading activation models (Collins \& Loftus, 1975; Collins \& Quillian, 1969, 1972; Glass \& Holyoak, 1975), do not readily 
lend themselves to representing more abstract concepts. There is no intuitively obvious hierarchy of sets into which such concepts can be placed.

In attempting to investigate abstract concepts, the present investigation adopts a featural approach, since this approach seems to provide a useful account of the observed characteristics of concrete concepts. It also makes relatively few assumptions about the relations between concepts and concerns itself mainly with what Rosch (1975) terms the "internal structure" of concepts.

The procedure adopted to investigate the concepts is therefore taken from the previous investigation of concrete categories described above (Hampton, 1979). If abstract concepts share a structure similar to that of concrete ones, then the number of category features that an instance of an abstract concept possesses should be a good predictor of the degree to which it is regarded as a typical example of that concept. In the first experiment, concept definitions for eight abstract concepts were elicited. In the second experiment, examples were elicited and assigned to four levels of category membership. The third study then tested the extent to which the number of concept features possessed by an example predicted its membership in the concept.

\section{EXPERIMENT 1}

The aim of this experiment was to obtain feature definitions for eight abstract terms from subjects' descriptions of the important characteristics of those concepts.

\section{Method}

Subjects. Thirty-two undergraduates at University College London acted as volunteer paid subjects. Both males and females were used, and all subjects were British speakers of English.

Materials. Eight concepts were selected for investigation. All except one had as referents things that are not physical, concrete objects. Furthermore, the concepts were selected on the basis that a set of examples of the concepts could be produced with reasonable ease to form a category of instances, as required by the procedure. The concepts selected were a work of art, a belief, a crime, a just decision, an instinct, a rule, a science, and a kind of work.

Procedure. Questionnaire forms were given to the subjects, who completed them without a time limit. The form gave the list of eight concepts and asked subjects to list on separate sheets anything they felt might be important in determining whether something was correctly described by each term. Subjects were also encouraged to consider why some examples of the concepts might be more or less typical, as a strategy for discovering relevant features. The order of the concepts was balanced so that each occurred in each ordinal position the same number of times and also followed each of the other concepts the same number of times.

\section{Results}

The responses made by each subject were tabulated according to their frequency across subjects. Appen$\operatorname{dix}$ A shows the most common responses for each of the eight abstract words, together with their production frequency $(\mathrm{PF})$.

\section{EXPERIMENT 2}

The aim of the second experiment was to provide for each concept a set of examples of varying degrees of category membership, to allow the polymorphous concept model to be tested. One difference between the concrete terms used by Hampton (1979) and those used in the present investigation is that whereas the concrete terms were categories of nameable objects or activities, some of the abstract terms, such as "a just decision," refer to an openended set of actual or hypothetical situations, which need not be nameable by a single word. It was therefore necessary to generate examples for each of the abstract concepts in order that the relationship between the number of category features they possessed and their degree of category membership could be ascertained. Furthermore, the examples had to be assigned a position on a scale, indicating their degree of category membership. Four levels were distinguished on this scale. The top two levels were for typical and atypical category exemplars, respectively, whereas the third and fourth levels were for related nonmembers and unrelated nonmembers of the category.

The experiment involved, first, the generation of suitable examples and then the selection and assignment of examples to the above four levels of category membership.

\section{Method}

Subjects. Nine subjects were used to generate examples. They were students and postgraduates at University College London, and they volunteered to participate. Five judges were asked to assign examples to levels of category membership. The judges were postgraduate students also at the University College London.

Procedure. A first group of subjects were given a questionnaire to fill in, in their own time. For each of the eight abstract terms, they were encouraged to give more than one example at each level if they wished. On the basis of the examples obtained a group of judges considered the examples independently, select ing four items for each of four levels: good examples, atypical examples, related nonexamples, and unrelated nonexamples. These levels will be termed $A, B, C$, and $D$, respectively. The instructions were to pick items that best illustrated each level of membership, on the basis of the generally accepted notions of the concept in question. The examples were typed out in approximately alphabetic order, so that no information about the class in which an item had been produced was available to the judges.

After making their selections, the judges were asked to rate the difficulty experienced with each concept on a 5-point scale. From the examples selected by the five judges, a final selection of four items for each membership level was made by the author on the basis of the following criteria: (1) Select the most frequently chosen items for a level, as long as they were not chosen equally often for a neighboring level, (2) choose those items that were selected least often for other levels, (3) for the final choice between remaining alternatives, choose those items that are most specific and avoid items that are ambiguous or are merely descriptions of the concept.

\section{Results and Discussion}

Apart from occasional lapses, the subjects generating examples understood the task well and produced some creative responses. In particular, many of the responses 
appeared to reflect the subject's personal experience with examples of the concept. For instance, in the case of the concept "rule," the most commonly encountered rules appeared to be in schools, driving, and in the cinema or other public places. There seemed to be at least two ways in which a nonexample could be provided; one was to find an antonym within the same, more general concept (e.g., philanthropy as opposed to crime, or ugliness as opposed to art). The alternative was to produce a completely unrelated word, which rather than contradicting the concept, is simply anomalous (e.g., a calendar as a kind of work, or a pimple as a just decision). These different strategies reflect the fact that the contrast set of a concept is not always specified in an unambiguous way. This is particularly apparent in the case of the concept "a belief," for which some subjects seemed to be giving examples that differed in the extent to which they were justifiable beliefs, as if an irrational belief was no longer a belief. If a contrast set such as "fact" had been suggested to subjects, then clearer data could have been obtained. It is perhaps interesting that the number of examples offered by more than one subject varied between the four levels of membership. Repeated responses were given far more frequently for the good examples ( 24 repetitions) than for any of the other three classes, which suggests that there may be some more or less prototypical examples of these concepts (such as chemistry for science, or murder for crime) that can be easily retrieved and are the first items that come to mind for different people.

Following the selection of four items for each of the membership levels, there were 16 examples for each of the concepts. These examples are shown in Appendix B.

The mean rated difficulty for each concept is given and discussed in a later section.

\section{EXPERIMENT 3}

The aim of this final stage of the investigation was to bring together the results of Experiments 1 and 2, in order to test the polymorphous concept model for the abstract terms under consideration. The model predicts that the degree to which an example is a member of the category (which in the present case refers to the level of category membership to which an example was assigned in Experiment 2) should be predictable from the number of category features that the example possesses. (These were the features elicited in Experiment 1.) The aim of this final experiment was therefore to assess the degree to which each of the chosen examples possesses each of the features of the abstract concept, by obtain. ing ratings from a group of subjects. The success of the prediction made by the polymorphous concept model can then be assessed.

\section{Method}

Subjects. Sixteen undergraduate psychology students at University College London acted as subjects.
Procedure. Following a procedure similar to that used by Hampton (1979), the examples were typed out in approximately alphabetical order as rows in a matrix, across the top of which were ranged the features of the concepts produced in Experiment 1 (see Appendix A). In order to limit the number of features to a manageable number, a selection criterion was applied. The features were arranged in order of production frequency, and the first 12 features were included regardless of how many subjects had given the 12 th feature. Then more features were included, either to the frequency level at which at least 15 features were included or the frequency level at which only four subjects gave a feature, whichever constraint applied first. By this means, each concept was assigned between 12 and 16 features. These are shown in Appendix A. These features were written opposite each of the columns in the matrix. Each subject was given two concept matrices to complete, so that each concept was judged by four subjects. Sixteen different pairings of the concepts were used, and any one concept occurred equally often in the first or second position. The subjects' task was to fill in the matrices by putting a number from 2 to -2 in each cell, according to whether the feature was true of the example or not. The subjects were instructed to place a -2 in a cell if the feature either was false for the example or was inapplicable and made no sense when applied to the example. The subjects were run in two group sessions and took approximately $30 \mathrm{~min}$ to complete the two matrices.

\section{Results and Discussion}

The ratings of the four judges for each concept were summed to yield a composite matrix, with entries taking a range of possible values from +8 to -8 (to be referred to as feature scores). Agreement between subjects was reasonably good. For instance, across concepts, $23 \%$ of the feature scores had values of +8 or -8 , indicating unanimous agreement of the judges. From the matrices, a measure of feature overlap for the examples was obtained by summing the feature scores for each example. If the polymorphous concept gives a good definition of these abstract terms, then there should be little or no overlap of the sum of feature scores between the examples at different levels, and the sum of feature scores of the levels should be ordered A, B, C, D. Table 1 shows the membership level of each example, ranked according to the sum of feature scores of that example so that the degree of overlap between levels may be easily seen. A measure of the degree of overlap is also given in the table; this is the number of transpositions of neighboring items required to reduce the overlap to zero. For three of the concepts, "a work of art," "a science," and "a crime," the fit of the definition was good (overlap of 3 or less). "A kind of work" and "a just decision" were also reasonably well fitted by the definition (overlaps of 6 and 7). All of these concepts showed significant differences between the four membership levels at the .0005 level on a Jonckeere test (Leach, 1979). However, the remaining three concepts "a rule," "a belief," and "an instinct," were not so well fitted, although there was still a significant trend in the predicted direction at the .01 level on the Jonckeere test.

The reasons for the less than perfect fit of the polymorphous model to these concepts may come from various sources. One possible reason is that these concepts 
Table 1

Examples of Each Abstract Concept Listed in Order of Sum of Feature Scores (SFS) and Labeled According to Typicality Class

\begin{tabular}{|c|c|c|c|c|c|c|c|c|c|c|c|c|c|c|c|c|c|}
\hline \multirow[b]{2}{*}{ Concept } & \multicolumn{16}{|c|}{ Order of Examples by SFS } & \multirow[b]{2}{*}{$\mathrm{O}$} \\
\hline & 1 & 2 & 3 & 4 & 5 & 6 & 7 & 8 & 9 & 10 & 11 & 12 & 13 & 14 & 15 & 16 & \\
\hline A Science & $\mathbf{A}$ & A & B & A & A & B & B & B & $\mathrm{C}$ & $\mathrm{C}$ & $\mathrm{C}$ & $\mathrm{C}$ & $\mathrm{D}$ & D & $\mathrm{D}$ & D & 2 \\
\hline A Work of Art & A & A & A & A & B & B & B & $\mathrm{C}$ & $\mathrm{C}$ & $\mathrm{C}$ & B & $\mathrm{C}$ & $\mathrm{D}$ & D & $\mathrm{D}$ & D & 3 \\
\hline A Crime & $\mathrm{A}$ & A & A & $A$ & $\mathrm{~B}$ & B & B & C & $\mathrm{C}$ & B & $\mathrm{C}$ & $\mathrm{D}$ & $\mathrm{C}$ & $\mathrm{D}$ & $\mathrm{D}$ & D & 3 \\
\hline A Kind of Work & A & $\mathrm{A}$ & B & A & A & B & B & $\mathrm{B}$ & D & $\mathrm{C}$ & $\mathrm{C}$ & $\mathrm{C}$ & $\mathrm{C}$ & D & D & D & 6 \\
\hline A Just Decision & A & A & A & A & B & B & $\mathrm{C}$ & B & C & B & $\mathrm{D}$ & $\mathrm{C}$ & $\mathrm{D}$ & D & C & D & 7 \\
\hline A Belief & A & A & $\mathrm{B}$ & A & $\mathrm{C}$ & A & $\mathrm{C}$ & B & C & B & B & D & C & D & $\mathrm{D}$ & D & 12 \\
\hline An Instinct & $\mathrm{B}$ & A & B & A & $\mathrm{C}$ & B & A & $\mathrm{C}$ & A & $\mathrm{C}$ & B & $\mathrm{C}$ & D & $\mathrm{D}$ & $\mathrm{D}$ & D & 16 \\
\hline A Rule & B & B & B & A & $\mathrm{C}$ & A & B & A & $\mathrm{C}$ & A & D & $\mathrm{C}$ & $\mathrm{C}$ & $\mathrm{D}$ & $\mathrm{D}$ & D & 21 \\
\hline
\end{tabular}

Note $-A=$ typical example,$B=$ atypical example, $C=$ related nonexample,$D=$ unrelated nonexample; $O=$ degree of overlap.

are not defined polymorphously, but in terms of conjunctive or disjunctive sets of defining properties. This possibility receives little support from the data. Of the eight concepts, only "science" could be given a conjunctive definition in terms of necessary features, and this concept was also well fitted by a polymorphous definition as regards membership. In no case could an adequate disjunction of features be found as a definition. However, the possibility remains open that there is no one definition for concepts such as "a rule," but rather a number of different concepts each with its own definition; in particular, the reversal of the typicality classes $\mathrm{A}$ and $\mathrm{B}$, for the concept RULE, suggests that typicality may not be defined in terms of similarity to a prototype (as found in Rosch's, 1975, experiments), but rather in terms of the frequency with which examples are encountered.

Other possible sources of the lack of fit may be inherent in the procedure, for instance, the choice of examples for Levels A-D and the assignment of the features to those examples. Differential difficulty with these two tasks might account for the different degrees of overlap found in different concepts. To investigate this possibility, a correlation was calculated between the degree of overlap between membership levels and the rated difficulty of choosing examples for the four levels, which was obtained for each concept from the five judges who selected the examples. These two variables were correlated across concepts at a significant level (Spearman's rho $=.786, N=8, p<.05$ ). Thus the concepts for which it was easy to choose examples were also those that showed a good fit on the polymorphous definition. Table 2 gives the degree of overlap and the mean rated difficulty of choosing examples for each concept.

An alternative reason for the lack of fit may lie in the assignment of feature scores to the examples. A possible measure of the difficulty of this task may be the frequency of feature scores that lie within the midrange of +4 to -4 , indicating either disagreement between the judges or lack of confidence concerning the possession of a particular feature. Table 2 also shows the mean number of feature scores lying in the midrange for each concept matrix. A second correlation was then calculated between the degree of overlap and this measure. This correlation was also significant (Spearman's tho $=$ $.649, \mathrm{~N}=8, \mathrm{p}<.05$ ). There was no significant correlation between the rated difficulty of choosing examples and the number of midrange feature scores (Spearman's rho $=.244, \mathrm{~N}=8$ ). This implies that these two measures indicate independent explanations of why some concepts are better characterized by a polymorphous concept.

A final possible source of error contributing to the lack of fit of some concepts lies in the original elicitation of properties in Experiment 1. If the difficulty of producing a definition of the concept is contributing to the lack of fit, then there should be a significant positive correlation between the goodness of fit and some measure of the ease of producing properties to define the concept. A Spearman nonparametric correlation was calculated between the degree of overlap in the final fit and the total number of properties produced by all subjects (counting tokens, not types), across each category. The latter measure is shown in the final column of Table 2. This correlation was significant (Spearman's rho $=.804, \mathrm{~N}=8, \mathrm{p}<.05$ ). Furthermore, there was no significant correlation between the number of different types of feature produced for each concept and the

Table 2

Variables Associated With the Goodness of Fit of the Definitions of the Abstract Concepts

\begin{tabular}{lrccc}
\hline \multicolumn{1}{c}{ Concept } & O & D & FS & P \\
\hline A Science & 2 & 2.2 & 4.80 & 8.3 \\
A Work of Art & 3 & 2.6 & 6.20 & 6.1 \\
A Crime & 3 & 2.6 & 4.50 & 8.2 \\
A Kind of Work & 6 & 2.0 & 8.00 & 8.2 \\
A Just Decision & 7 & 4.0 & 7.25 & 5.1 \\
A Belief & 12 & 3.6 & 8.70 & 4.9 \\
An Instinct & 16 & 3.8 & 7.00 & 4.5 \\
A Rule & 21 & 4.2 & 7.40 & 5.2 \\
\hline
\end{tabular}

Note-Concepts are shown in order of degree of overlap $(O)$ of the typicality classes, $A-D . D=$ rated difficulty of choosing examples $(1=$ easy, $S=$ difficult; $F S=$ number of midrange feature scores per feature, maximum $=16 ; P=$ mean number of properties per subject. 
Table 3

Spearman's Nonparametric Correlation Coefficient $r_{s}$

\begin{tabular}{llll} 
& $\mathrm{D}$ & $\mathrm{FS}$ & $\mathrm{P}$ \\
\hline $\mathrm{O}$ & $.786^{*}$ & $.649^{*}$ & $-.804^{*}$ \\
$\mathrm{D}$ & & .244 & $-.732^{*}$ \\
FS & & & -.482 \\
\hline
\end{tabular}

Note $-N=8 . O=$ degree of overlap, $D=$ difficulty of choosing examples, $F S=$ number of midrange feature scores, $P=$ mean number of properties produced.

*Significant at 05 level.

\section{Table 4}

\begin{tabular}{lrrrrr} 
& \multicolumn{2}{c}{$\begin{array}{c}\text { Defining } \\
\text { Features }\end{array}$} & & \multicolumn{2}{c}{$\begin{array}{c}\text { Characteristic } \\
\text { Features }\end{array}$} \\
\cline { 2 - 3 } \cline { 5 - 6 } Concept & Mean PF & $\mathrm{N}$ & & Mean PF & $\mathrm{N}$ \\
\hline A Work of Art & 12.25 & 4 & 6.55 & 11 \\
A Belief & 4.67 & 3 & & 10.00 & 12 \\
A Crime & 14.36 & 11 & 6.25 & 4 \\
A Just Decision & 18.50 & 2 & 6.00 & 14 \\
An Instinct & 7.00 & 3 & 8.44 & 9 \\
A Rule & 10.00 & 5 & 7.00 & 7 \\
A Science & 7.33 & 12 & 13.33 & 3 \\
A Kind of Work & 14.57 & 7 & 9.25 & 8 \\
Combined & 11.04 & 47 & 7.94 & 68 \\
\hline
\end{tabular}

degree of overlap. It may therefore be concluded that the degree of overlap is associated with the general ease of producing features to define the concept, but not with the variety of different features produced. Table 3 shows the correlations among the measures associated with the degree of overlap.

To conclude, there are three measures associated with the goodness of fit of the polymorphous definitions. Following the order in which they appear in the procedure, these measures are: (1) the general ease of producing descriptive features, in terms of number, not variety, (2) the ease with which examples may be selected for testing at four levels of membership, ranging from good members to definite nonmembers, (3) the number of feature scores assigned to the features and examples that show a high degree of confidence and agreement between the judges.

Definingness of properties. The example-feature matrices allow an analysis to be made of the extent to which each feature in a concept's definition is "defining" or important. Smith et al. (1974) suggest that category features can be placed on a continuum of "definingness." Although disagreeing with Smith et al. in other respects, Hampton (1979) confirmed that features that are common to all members of a category, which Smith et al. term defining features, are produced more frequently than those that are possessed only by the typical category members, which are termed characteristic features. A similar analysis was made on the present data. The mean production frequencies for features common to all eight concept members and for features not possessed by all members was calculated for each concept. The results are shown in Table 4 . The defining features were produced on average with a higher frequency (mean $=$ 11.04) than were the characteristic features (mean = 7.94). The difference was significant at the .01 level on a $t$ test $[t(113)=2.82]$. This result may be taken as evidence that the abstract concepts do in some respects have a structure similar to the concrete noun categories studied by Hampton (1979). (The features common to all category members are asterisked in Appen$\operatorname{dix}$ A.)

\section{GENERAL DISCUSSION}

The results suggested that some abstract concepts could be well defined by a polymorphous concept but others less so. It was also shown that the degree to which a concept was well fitted by a polymorphous definition correlated positively with the ease of producing properties and of choosing examples and correlated negatively with the amount of disagreement or lack of confidence in the judges who assigned the feature scores to those examples. The conclusion may therefore be drawn that the concepts that do not yield a good fit give trouble in all stages of the test; the lack of fit is associated with a general difficulty in applying the method to these concepts, making the assessment of the typicality of their examples and the assignment of features to these examples a difficult task.

If the present abstract examples are compared with the concrete categories studied by Hampton (1979), a number of similarities and differences can be shown. In terms of similarity, both sets of concepts showed a dimension of definingness for their properties, such that the features common to all category examples were produced more frequently than others. However, the differences are perhaps more interesting. First, unlike many concrete categories such as "fruit" or "fish," the membership of abstract concepts is almost unlimited in the range of possible new instances that could be discovered or invented. It thus appears that abstract concepts may show greater freedom for all possible combinations of their features to occur in the real world. This independence between features may reduce what Rosch (1975) terms the "correlational structure of the world" for abstract concepts and hence weaken any prototype structure they may show. Second, the types of information involved in defining abstract concepts are obviously different from those involved in defining concrete ones. An intuitive analysis would indicate the common structure of the terms studied here as involving some kind of act or behavior, together with details of the agent, the motive, the effect, and social meaning of the action. The featural approach may then break down, because the relationship between features may be crucial and may constitute a higher order type of featural information that would greatly increase the complexity of the concept's structure.

The success of the polymorphous definitions of art, science, and crime suggests that these concepts do have a 
polymorphous structure, centered around a prototype, similar to more concrete categories such as "fruit" and "furniture." Given a more thorough method for obtaining the concept properties and for assessing the typicality of examples, it is perhaps possible that the other concepts would begin to show a similar structure. No obvious alternative structure for the definition, such as a conjunction or disjunction of features, was evident in the data for these concepts. However, it must be accepted that the generality of the polymorphous concept model for abstract concepts has not been convincingly dem. onstrated.

It is interesting to note that the best fit concepts were those for which it is perhaps more easy to produce a standard list of category members. "“Crime" and "science" may be found as categories in Battig and Montague's, 1969, category norms.) However, one might also expect that it would be relatively easy to generate examples for "instinct", and yet the fit was not good. It is possible that the two concepts "belief" and "rule" are not best thought of as categories at all. Thus, while it is possible for any concept to delimit a class of things to which the concept name applies and for someone then to search for a property definition that will account for the membership of that class, there may be some concepts for which this method of analysis is not appropriate.

Further research is needed to develop alternative views of how the semantics of such abstract concepts should be represented. The extension of semantic memory research into this area is an important way in which the relevance of the theoretical models to a wider world can be tested. In particular, the data suggest that the generality of existing models of concept structure may be restricted. Thus, discovering the boundary conditions of such models represents a goal for future research.

\section{REFERENCES}

Battig, W. F., \& Montague, W. E. Category norms for verbal items in 56 categories: A replication and extension of the Connecticut category norms. Journal of Experimental Psychology Monograph, 1969, 80(3, Pt. 2).

Collins, A. M., \& Loftus, E. F. A spreading activation theory of semantic processing. Psychological Review, 1975, 82, 407428.

Collins, A. M., \& Quillian, M. R. Retrieval time from semantic memory. Journal of Verbal Learning and Verbal Behavior, $1969,8,240-247$.

Collins, A. M., \& Quillian, M. R. How to make a language user. In E. Tulving \& W. Donaldson (Eds.), Organization of memory. New York: Academic Press, 1972.

Glass, A. L., \& HolyoaK, K. J. Alternative conceptions of semantic memory. Cognition, 1975, 3, 313-339.

Hampton, J. A. Polymorphous concepts in semantic memory. Journal of Verbal Learning and Verbal Behavior, 1979, 18, 441-461.

$\mathrm{LEACH}, \mathrm{C}$. Introduction to statistics. A non-parametric approach to the social sciences. Chichester, England: Wiley, 1979.

McCloskey, M. E., \& Glucksberg, S. Decision processes in verifying category membership statements: Implications for models of semantic memory. Cognitive Psychology, 1979, 11, 1-37.

Rosch, E. Cognitive representations of semantic categories. Journal of Experimental Psychology: General, 1975, 104, 192-233.

Rosch, E., \& Mervis, C. B. Family resemblances: Studies in the internal structure of categories. Cognitive Psychology, 1975 , 7, 573-605.

Smith, E. E., Shoben, E. J., \& Rips, L. J. Structure and process in semantic memory: A featural model for semantic decisions. Psychological Review, 1974, 81, 214-241.

Appendix A

Feature Definitions of the Eight Abstract Concepts

\begin{tabular}{|c|}
\hline Property \\
\hline A Work of Art \\
\hline 1. Is done, made \\
\hline 2. Is visual \\
\hline 3. Is pleasing \\
\hline 4. Is man-made \\
\hline 5. Is beautiful \\
\hline 6. Is an object \\
\hline 7. Involves skill \\
\hline 8. Is meaningful \\
\hline 9. Is original \\
\hline 10. Is an expression \\
\hline 11. Is expensive \\
\hline 12. Its value endures over time \\
\hline 13. Contains message \\
\hline 14. Arouses some emotion \\
\hline 15. Is publicly acclaimed \\
\hline
\end{tabular}

\begin{tabular}{lc}
\multicolumn{1}{c}{ A Belief } \\
1. Is something human & 32 \\
2. Is something held to be true & 15 \\
3. Personal & 12 \\
4. Not based on evidence & 9 \\
5. Life style can be based on it & 9 \\
6. Religious & 8 \\
7. Held rigidly & 8 \\
8. Is a faith & 7 \\
9. Is not susceptible to proof & 6 \\
10. Is taught and learnt & 6 \\
11. Not necessarily logical & $6 *$ \\
12. Not proven & $4 *$ \\
13. Is based on available evidence & $4^{*}$ \\
14. Is taught by others & 4 \\
15. Is strong & 4
\end{tabular}

1. An act or deed 24*

2. Has some adverse effect on some victim $24^{*}$

3. Breaks some law or rule 23*

4. Breaks a statutory law $14^{*}$

5 . Is done deliberately $13^{*}$

6. Breaks some moral or social code $13^{*}$

7. Has an adverse effect on other individuals $12^{*}$

8. Has an adverse effect on society 11*

9. Harms or hurts $11^{*}$

10. Is punishable

11. Is judged relative to a society

12. Is done by an individual

13. Is done against property

14. Is antisocial

15. Causes injury

\section{A Just Decision}

1. Is made by someone

2 . Is supported by the majority opinion

3. Is fair 


Property
4. Is impartial
5. Is legal
6. Punishes wrong
7. Takes account of all views
8. Is a judgment
9. Gives due desserts
10. Takes account of all of the facts
11. Is based on moral principles
12. Is based on the maker's personal view
13. Is fair to all
14. Is unemotional
15. Is socially and culturally acceptable
16. Maximizes the general benefit

An Instinct

\section{Is innate, inborn}

2. Is au tomatic, unconscious

3. Is an action or behavior

4. Is not logical

5 . Is for self-preservation

6 . Is a response, reaction

7. Is most obvious in animals

8. Is a feeling

9. Is present in a whole species

10. Is unpremeditated

11. Is a sixth sense

12. Is found in animals or humans

A Rule

1. Is something followed

2. Is followed by someone

3. Can be broken

4. Made by some group of people

5. About Behavior

6. For social reasons

7. Entails punishment or censure if broken

8. Is followed by all to whom it applies

9. Is a method

10. Is about morals

11. Is made by an authority

12. Is for protecting the safety of others

\section{A Science}

1. Is about natural physical facts

2. Involves experiments

3. Involves testing

4. Is a study

5. Is objective, unemotional

6. Has applications

7. Involves observation

8. Is logical, deductive

9. Is mathematical, quantitative

10. Is about facts

11. Involves theories

12. Involves hypotheses

13. Is precise, accurate

14. Involves rules

15. Involves proving

A Kind of Work

1. Is done by someone

2. Uses up energy

3. Has some reward

4. Is that for which one obtains money

5 . Is done for some reward

6. Is physical

7. Is mental

8. Is done for payment

9. Requires effort

10. Is done to provide the necessities of life

11. Is time-consuming
PF

8

8

8

6

6

5

5

5*

4

4

4

4

19

17

$9 *$

9

7*

5

$5 *$

5

3

3

\begin{tabular}{ll}
\multicolumn{1}{c}{ Property } & PF \\
\hline 12. Is pleasurable & 6 \\
13. Is unpleasant & 6 \\
14. Is done for satisfaction & 6 \\
15. Is exhausting & $5^{*}$ \\
\hline
\end{tabular}

Note-Properties are listed in order of production frequency (PF) (out of 32). *Items possessed by all category members (see Experiment 3).

Appendix B

Examples Used for Testing Concept Definitions With Sum of Feature Scores (SFS)

$\begin{array}{cc}\text { Example } & \text { SFS } \\ \text { A Work of Art }\end{array}$

(A) The Mona Lisa 100

Henry Moore's sculpture

Leonardo's cartoons $\quad 82$

Paintings by Constable

(B) A totally red painting 25

Andy Warhol's Brillo boxes in a gailery

"King Lear"

Blenheim Palace 81

(C) An intricately decorated wedding cake 61

Battersea Power Station

The Eiffel Tower 58

New Covent Garden market 39

(D) A lump of earth $\quad-52$

A zip fastener $\quad-20$

A piece of string $\quad-42$

God $\quad-24$

A Belief

(A) Buddhism 91

Christianity 88

Communism 62

Life after death

(B) Evolution 17

In being able to change the weather by prayer 19

Patriotism 33

That Jesus Christ was the son of God $\quad 80$

(C) Atheism 56

Chauvinism 35

Health foods

"I am an agnostic" 26

(D) The alphabet 0

Deep-sea diving $\quad-28$

Eating $\quad-15$

Music 12

(A) Mugging $\quad 81$

Murder $\quad 85$

Rape $\quad 94$

Robbery $\quad 87$

(B) Antisemitism 61

Drinking and driving 75

Negligence of animals $\quad 43$

Treason 65

(C) Adultery 46

Doctor ignoring parking restrictions $\quad-\quad 9$

Drug addiction $\quad 45$

Going on the straight and narrow $\quad-45$

(D) A handbag $\quad-114$

Love -35

Pneumonia $\quad-56$

Sleep -83 


\section{A Just Decision}

(A) Giving the vote to women

61

Giving an obvious penalty in football

To free an innocent person

To protect the weak

(B) Declaration of war in 1939

The death sentence

Giving financial aid to a petty thief

Redistribution of wealth

(C) Tossing a coin to decide an argument Castration for rape

Decisions based on minority opinions Decisions involving rigged ballots

(D) A googly

Mass arrest and conviction without trial A pimple

To rob a poor man of his money

An Instinct

(A) An axolotl knows how to swim even if prevented from moving early in life Butterflies knowing how to fly Self-preservation Survival

(B) Aggressive attitude of a dog toward a cat Sex

To shake your head in disapproval To smile

(C) Anger

Getting on with children

Green fingers

Learning to do things

(D) $\mathrm{A}$ biro

A carpenter

Operating computers

Tightrope walking

\section{A Rule}

(A) No smoking in school

All dogs should be on leads on roads

Swearing is forbidden

School caps must be worn by boys until their 3rd year at school

(B) Ladies cannot enter the royal enclosure at Ascot wearing trousers

No lying on the floor in a cinema

No incest

Road signs
(C) Hooks Law of Gravity Apartheid

Anarchy

A chilblain

A bath

Being in love with someone very much Silencing people

A Science

(A) Biology 93

Chemistry 107

Physics 112

Zoology 94

(B) Economics 97

Geography 69

Linguistics 74

Mathematics $\quad 87$

(C) Advertising 3

English 6

History 22

Study of French

(D) Bus conductor

Ceiling $\quad-94$

Fruitcake $\quad-97$

Wall -81

A Kind of Work

(A) Being a bank clerk $\quad 70$

Farming 90

Manufacturing $\quad 75$

Road digging 71

(B) Artist who intends to sell his work 50

Learning 58

Postgraduate research $\quad 72$

Riding a bicycle uphill 41

(C) Cardiac massage 24

Just sitting 25

A person living a life of ease and contentment 11

Watching TV 5

(D) A building $\quad-57$

A calendar $\quad-76$

Death $\quad-19$

Sleeping

(Received for publication May 5, 1980; revision accepted July 28,1980 .) 